\title{
Exact Solutions of a Spatially Dependent Mass Dirac Equation for Coulomb Field plus Tensor Interaction via Laplace Transformation Method
}

\author{
M. Eshghi, ${ }^{1}$ M. Hamzavi, ${ }^{2}$ and S. M. Ikhdair ${ }^{3,4}$ \\ ${ }^{1}$ Physics Department, Imam Hossein Comprehensive University, Tehran, Iran \\ ${ }^{2}$ Department of Basic Sciences, Shahrood Branch, Islamic Azad University, Shahrood, Iran \\ ${ }^{3}$ Physics Department, Near East University, 922022 Nicosia, Northern Cyprus, Turkey \\ ${ }^{4}$ Physics Department, Faculty of Science, An-Najah National University, Nablus, West Bank, Palestine
}

Correspondence should be addressed to M. Eshghi, eshgi54@gmail.com

Received 9 October 2012; Accepted 4 November 2012

Academic Editor: Kingman Cheung

Copyright (C) 2012 M. Eshghi et al. This is an open access article distributed under the Creative Commons Attribution License, which permits unrestricted use, distribution, and reproduction in any medium, provided the original work is properly cited.

\begin{abstract}
The spatially dependent mass Dirac equation is solved exactly for attractive scalar and repulsive vector Coulomb potentials including a tensor interaction potential under the spin and pseudospin (p-spin) symmetric limits by using the Laplace transformation method (LTM). Closed forms of the energy eigenvalue equation and wave functions are obtained for arbitrary spin-orbit quantum number $\kappa$. Some numerical results are given too. The effect of the tensor interaction on the bound states is presented. It is shown that the tensor interaction removes the degeneracy between two states in the spin doublets. We also investigate the effects of the spatially-dependent mass on the bound states under the conditions of the spin symmetric limit and in the absence of tensor interaction $(T=0)$.
\end{abstract}

\section{Introduction}

Dirac equation has become one of the most appealing relativistic wave equations for spin- $1 / 2$ particles. However, solving such a wave equation is still a very challenging problem even if it has been derived more than 80 years ago and has been utilized profusely. It is always useful to investigate the relativistic effects [1-4]. For example, in the relativistic treatment of nuclear phenomena the Dirac equation is used to describe the behavior of the nuclei in nucleus and also in solving many problems of high-energy physics and chemistry. For this reason, it has been used extensively to study the relativistic heavy ion collisions, heavy ion spectroscopy, and more recently in laser-matter interaction (for a review, see [5] and references therein) and condensed matter physics [6]. 
On the other hand, systems with position-dependent mass (PDM) have been found to be very useful in studying the physical properties of various microstructures [7-14]. Recently, there has been increased interest in searching for analytical solutions of the Dirac equation with PDM and with constant mass under the spin and p-spin symmetries [15-34].

Here, we shall attempt to solve the Dirac equation by using the Laplace transform method (LTM). The LTM is an integral transform and is comprehensively useful in physics and engineering [35] and recently used by many authors to solve the Schrödinger equation for different potential forms [36-40]. This method could be a nearly new formalism in the literature and serve as a powerful algebraic treatment for solving the second-order differential equations. As a result, the LTM describes a simple way for solving of radial and onedimensional differential equations. The other advantage of this method is that a secondorder equation can be converted into more simpler form whose solutions may be obtained easily [36]. In this paper, we obtain solution of the Dirac equation both PDM and tensor interaction for attractive scalar and repulsive vector Coulomb potential under the spin and p-spin symmetry limits. We give some numerical results.

\section{Review to Dirac Equation including Tensor Coupling}

The Dirac equation which describes a nucleon in repulsive vector $V(r)$ and attractive scalar $S(r)$ and a tensor $U(r)$ potentials is written as

$$
\left[\vec{\alpha} \cdot \vec{p}+\beta\left(M(r) c^{2}+S(r)\right)-i \beta \vec{\alpha} \cdot \widehat{r} U(r)\right] \psi(\vec{r})=[E-V(r)] \psi(\vec{r}),
$$

where $M(r)$ is the effective mass of the fermionic particle, $E$ is the relativistic energy of the system, $\vec{p}=-i \hbar \vec{\nabla}$ is the three-dimensional momentum operator. $\vec{\alpha}$ and $\beta$ are the $4 \times 4$ Dirac matrices give as

$$
\vec{\alpha}=\left(\begin{array}{cc}
0 & \vec{\sigma} \\
\vec{\sigma} & 0
\end{array}\right), \quad \beta=\left(\begin{array}{cc}
I & 0 \\
0 & -I
\end{array}\right)
$$

where $I$ is $2 \times 2$ unitary matrix and $\vec{\sigma}$ are three-vector spin matrices

$$
\sigma_{1}=\left(\begin{array}{cc}
0 & 1 \\
1 & 0
\end{array}\right), \quad \sigma_{2}=\left(\begin{array}{cc}
0 & -i \\
i & 0
\end{array}\right), \quad \sigma_{3}=\left(\begin{array}{cc}
1 & 0 \\
0 & -1
\end{array}\right)
$$

The total angular momentum operator $\vec{J}$ and spin-orbit $K=(\vec{\sigma} \cdot \vec{L}+1)$, where $\vec{L}$ is orbital angular momentum, of the spherical nucleons commute with the Dirac Hamiltonian. The eigenvalues of spin-orbit coupling operator are $\kappa=(j+1 / 2)>0$ and $\kappa=-(j+1 / 2)<0$ for unaligned spin $j=l-1 / 2$ and the aligned spin $j=l+1 / 2$, respectively. $\left(H^{2}, K, J^{2}, J_{z}\right)$ can be taken as the complete set of the conservative quantities. Thus, the spinor wave functions can be classified according to their angular momentum $j$, spin-orbit quantum number $\kappa$ and the radial quantum number $n$ can be written as follows:

$$
\psi_{n \kappa}(\vec{r})=\left(\begin{array}{c}
f_{n \kappa}(\vec{r}) \\
g_{n \kappa}(\vec{r})
\end{array}\right)=\frac{1}{r}\left(\begin{array}{c}
F_{n \kappa}(r) Y_{j m}^{l}(\theta, \varphi) \\
i G_{n \kappa}(r) Y_{j m}^{\tilde{l}}(\theta, \varphi)
\end{array}\right)
$$


where $f_{n \kappa}(\vec{r})$ is the upper (large) component and $g_{n \kappa}(\vec{r})$ is the lower (small) component of the Dirac spinors. $Y_{j m}^{l}(\theta, \varphi)$ and $Y_{j m}^{\tilde{l}}(\theta, \varphi)$ are spin and p-spin spherical harmonics, respectively, and $m$ is the projection of the angular momentum on the $z$-axis. Substituting (2.4) into (2.1) and using the following relations [41] as

$$
\begin{gathered}
(\vec{\sigma} \cdot \vec{A})(\vec{\sigma} \cdot \vec{B})=\vec{A} \cdot \vec{B}+i \vec{\sigma} \cdot(\vec{A} \times \vec{B}), \\
(\vec{\sigma} \cdot \vec{P})=\vec{\sigma} \cdot \hat{r}\left(\hat{r} \cdot \vec{P}+i \frac{\vec{\sigma} \cdot \vec{L}}{r}\right),
\end{gathered}
$$

and with the following properties:

$$
\begin{aligned}
& \vec{\sigma} \cdot \vec{L}\left\{\begin{array}{l}
Y_{j m}^{\tilde{l}}(\theta, \phi) \\
Y_{j m}^{l}(\theta, \phi)
\end{array}=\left\{\begin{array}{l}
(\kappa-1) Y_{j m}^{\tilde{l}}(\theta, \phi), \\
-(\kappa-1) Y_{j m}^{l}(\theta, \phi),
\end{array}\right.\right. \\
& \vec{\sigma} \cdot \hat{r}\left\{\begin{array}{l}
Y_{j m}^{\tilde{l}}(\theta, \phi) \\
Y_{j m}^{l}(\theta, \phi)
\end{array}=\left\{\begin{array}{l}
-Y_{j m}^{l}(\theta, \phi), \\
-Y_{j m}^{\tilde{l}}(\theta, \phi),
\end{array}\right.\right.
\end{aligned}
$$

one obtains two coupled differential equations for upper and lower radial wave functions $F_{n \kappa}(r)$ and $G_{n \kappa}(r)$ as

$$
\begin{aligned}
& \left(\frac{d}{d r}+\frac{\kappa}{r}-U(r)\right) F_{n \mathcal{K}}(r)=\left(M(r) c^{2}+E_{n \mathcal{K}}-\Delta(r)\right) G_{n \mathcal{K}}(r), \\
& \left(\frac{d}{d r}-\frac{\mathcal{\kappa}}{r}+U(r)\right) G_{n \mathcal{K}}(r)=\left(M(r) c^{2}-E_{n \mathcal{K}}+\Sigma(r)\right) F_{n \mathcal{K}}(r),
\end{aligned}
$$

where

$$
\begin{aligned}
& \Delta(r)=V(r)-S(r), \\
& \Sigma(r)=V(r)+S(r) .
\end{aligned}
$$

Eliminating $F_{n \kappa}(r)$ and $G_{n \kappa}(r)$ from (2.5e) and (2.5f), we finally obtain the following two Schrödinger-like differential equations for the upper and lower radial spinor components, respectively:

$$
\begin{aligned}
& {\left[\frac{d^{2}}{d r^{2}}-\frac{\kappa(\kappa+1)}{r^{2}}+\frac{2 \kappa}{r} U(r)-\frac{d U(r)}{d r}-U^{2}(r)\right] F_{n \kappa}(r)} \\
& -\frac{d M(r) / d r-d \Delta(r) / d r}{M(r)+E_{n \kappa}-\Delta(r)}\left(\frac{d}{d r}+\frac{\kappa}{r}-U(r)\right) F_{n \kappa}(r) \\
& =\frac{1}{(\hbar c)^{2}}\left[\left(M(r) c^{2}+E_{n \kappa}-\Delta(r)\right)\left(M(r) c^{2}-E_{n \kappa}+\Sigma(r)\right)\right] F_{n \kappa}(r),
\end{aligned}
$$




$$
\begin{aligned}
& {\left[\frac{d^{2}}{d r^{2}}-\frac{\kappa(\kappa-1)}{r^{2}}+\frac{2 \kappa}{r} U(r)+\frac{d U(r)}{d r}-U^{2}(r)\right] G_{n \kappa}(r)} \\
& -\frac{d M(r) / d r+d \Sigma(r) / d r}{M(r)-E_{n \kappa}+\Sigma(r)}\left(\frac{d}{d r}-\frac{\kappa}{r}+U(r)\right) G_{n \kappa}(r) \\
& =\frac{1}{(\hbar c)^{2}}\left[\left(M(r) c^{2}+E_{n \kappa}-\Delta(r)\right)\left(M(r) c^{2}-E_{n \kappa}+\Sigma(r)\right)\right] G_{n \kappa}(r),
\end{aligned}
$$

where $\kappa(\kappa-1)=\tilde{l}(\tilde{l}+1)$ and $\kappa(\kappa+1)=l(l+1)$. These radial wave functions are required to satisfy the necessary boundary conditions. The spin-orbit quantum number $\kappa$ is related to the quantum numbers for spin symmetry $l$ and p-spin symmetry $\tilde{l}$ as

$$
\mathcal{K}= \begin{cases}-(l+1)=-\left(j+\frac{1}{2}\right),\left(s_{1 / 2}, p_{3 / 2}, \text { etc. }\right) & j=l+\frac{1}{2}, \text { aligned spin }(\kappa<0), \\
l=j+\frac{1}{2},\left(p_{1 / 2}, d_{3 / 2}, \text { etc. }\right) & j=l-\frac{1}{2}, \text { unaligned spin }(\kappa>0),\end{cases}
$$

and the quasi-degenerate doublet structure can be expressed in terms of a p-spin angular momentum $\widetilde{s}=1 / 2$ and pseudo-orbital angular momentum $\tilde{l}$, which is defined as

$$
\mathcal{K}= \begin{cases}-\tilde{l}=-\left(j+\frac{1}{2}\right) ;\left(s_{1 / 2}, p_{3 / 2}, \text { etc. }\right) & j=\tilde{l}-\frac{1}{2}, \text { aligned p-spin }(\kappa<0), \\
\tilde{l}+1=j+\frac{1}{2} ;\left(d_{3 / 2}, f_{5 / 2}, \text { etc. }\right) & j=\tilde{l}+\frac{1}{2}, \text { unaligned p-spin }(\kappa>0),\end{cases}
$$

where $\kappa= \pm 1, \pm 2, \ldots$. For example, $\left(1 s_{1 / 2}, 0 d_{3 / 2}\right)$ and $\left(1 p_{3 / 2}, 0 f_{5 / 2}\right)$ can be considered as p-spin doublets. In the next section, we will consider the spin and p-spin symmetry cases.

\section{Relativistic Bound State Solutions}

\subsection{Spin Symmetry Case}

Equation (2.5i) cannot be solved analytically because of the last term $((d M(r) / d r-$ $\left.d \Delta(r) / d r) /\left(M(r)+E_{n \kappa}-\Delta(r)\right)\right)(d / d r+\kappa / r-U(r))$. Therefore, in solving the mathematical relation $d M(r) c^{2} / d r=d \Delta(r) / d r=d V(r) / d r$ [42,43], we can then exactly solve (2.5i). In this stage, we take the vector potential in the form of an attractive Coulomb-like field [18] as

$$
\Sigma(r)=V(r)=-\frac{\hbar c q_{v}}{r}, \quad q_{v}=q, r \neq 0
$$


where $q_{v}$ is being a vector dimensionless real parameter coupling constant and $\hbar c$ is being a constant with $\mathrm{J} \cdot \mathrm{fm}$ dimension. Also, it is convenient to take the mass function [18] as

$$
M(r)=m_{0}+\frac{m_{1}}{r}, \quad m_{1}=m_{0} \lambda_{0} b, \lambda_{0}=\frac{\hbar}{m_{0} c},
$$

where $m_{0}$ and $m_{1}$ stand for the rest mass of the fermionic particle and the perturbed mass, respectively. Further, $b$ is the dimensionless real constant to be set to zero for the constant mass case and $\lambda_{0}$ is the Compton-like wavelength in fm units. Further, the tensor interaction takes the simple form:

$$
U(r)=-\frac{T}{r}, \quad T=\frac{Z_{a} Z_{b} e^{2}}{4 \pi \varepsilon_{0}}, r \geq R_{c},
$$

where $R_{c}$ is the coulomb radius, $Z_{a}$ and $Z_{b}$ stand for the charges of the projectile particle $a$ and the target nucleus $b$, respectively.

Substituting (3.1)-(3.3) into (2.5i) considering the spin symmetry case where $\Delta(r)=$ $C_{s}=$ const., that is, $(d \Delta(r) / d r=0)[44,45]$. Thus, the equation obtained for the upper component of the Dirac spinor $F_{n k}(r)$ becomes

$$
\begin{aligned}
& \left\{\frac{d^{2}}{d r^{2}}-\frac{(\kappa+T)(\kappa+T+1)+b(b-q)}{r^{2}}+\frac{1}{\hbar c} \frac{\left[q\left(m_{0} c^{2}+E_{n \kappa}-C_{s}\right)+b\left(C_{s}-2 m_{0} c^{2}\right)\right]}{r}\right. \\
& \left.-\frac{1}{(\hbar c)^{2}}\left(m_{0} c^{2}-E_{n \kappa}\right)\left(m_{0} c^{2}+E_{n \kappa}-C_{s}\right)\right\} F_{n \kappa}(r)=0 .
\end{aligned}
$$

Further, defining the new parameters

$$
\begin{aligned}
& \lambda^{2}=(\kappa+T)(\kappa+T+1)+b(b-q), \\
& \delta^{2}=-\frac{1}{\hbar c}\left[q\left(m_{0} c^{2}+E_{n \kappa}-C_{s}\right)+b\left(C_{s}-2 m_{0} c^{2}\right)\right], \\
& \varepsilon^{2}=\frac{1}{(\hbar c)^{2}}\left(m_{0} c^{2}-E_{n \kappa}\right)\left(m_{0} c^{2}+E_{n \kappa}-C_{s}\right),
\end{aligned}
$$

and introducing $F_{n \kappa}(r)=r^{1 / 2} \varphi(r)$, then (3.4) turns into the form

$$
\begin{gathered}
{\left[r^{2} \frac{d^{2}}{d r^{2}}+r \frac{d}{d r}-r^{2}\left(\frac{v^{2}}{r^{2}}+\frac{\delta^{2}}{r}+\varepsilon^{2}\right)\right] \varphi(r)=0,} \\
v^{2}=\left(\kappa+T+\frac{1}{2}\right)^{2}+b(b-q) .
\end{gathered}
$$


Setting $\varphi(r)=r^{\alpha} f(r)$ with $\alpha$ is a constant and then inserting into (3.6a), we have

$$
\left[r^{2} \frac{d^{2}}{d r^{2}}+(2 \alpha+1) r \frac{d}{d r}-\left(v^{2}+\delta^{2} r+\varepsilon^{2} r^{2}-\alpha^{2}\right)\right] f(r)=0 .
$$

Now, to obtain a finite wave function when $r \rightarrow \infty$, if we take $\alpha=-v$ in (3.7) then it becomes

$$
\left[r \frac{d^{2}}{d r^{2}}-(2 v-1) \frac{d}{d r}-\left(\delta^{2}+\varepsilon^{2} r\right)\right] f(r)=0
$$

The LTM $[46,47]$

$$
L\{g(\rho)\}=f(t)=\int_{0}^{\infty} e^{-t \rho} g(\rho) d \rho
$$

leads to an equation

$$
\left(t^{2}-\varepsilon^{2}\right) \frac{d f(t)}{d t}+\left[(2 v+1) t+\delta^{2}\right] f(t)=0
$$

Equation (3.10) is a first-order differential equation and therefore we may directly make use of the integral to get the expression

$$
f(t)=N(t+\varepsilon)^{-(2 v+1)}\left(\frac{t-\varepsilon}{t+\varepsilon}\right)^{-\left[\delta^{2}+(2 v+1) \varepsilon\right] / 2 \varepsilon}
$$

where $N$ is a constant. Noting that $((t-\varepsilon) /(t+\varepsilon))^{-\left(\delta^{2}+(2 v+1) \varepsilon\right) / 2 \varepsilon}$ is a multi-valued function and the wave functions are required to be single-valued, we must take

$$
-\frac{\delta^{2}}{2 \varepsilon}-\frac{2 v+1}{2}=n, \quad n=0,1,2,3, \ldots
$$

which gives single-values wave functions. Using this requirement and further expanding (3.11) into series, we obtain

$$
f(t)=N^{\prime} \sum_{k=0}^{n} \frac{(-2 \varepsilon)^{k} n !}{(n-k) ! k !}(t+\varepsilon)^{-(2 v+1+k)}
$$

where $N^{\prime}$ is a constant. In terms of a simple extension of the inverse Laplace transformation $[46,47]$, we can immediately obtain

$$
f(r)=N^{\prime \prime} r^{2 v} e^{-\varepsilon r} \sum_{k=0}^{n} \frac{(-1)^{k} n !}{(n-k) ! k !} \frac{\Gamma(2 v+1)}{\Gamma(2 v+1+k)}(2 \varepsilon r)^{k},
$$


and from $\varphi(r)=r^{\alpha} f(r)$, we then obtain

$$
\varphi(r)=N^{\prime \prime \prime} r^{v} e^{-\varepsilon r} \sum_{k=0}^{n} \frac{(-1)^{k} n !}{(n-k) ! k !} \frac{\Gamma(2 v+1)}{\Gamma(2 v+1+k)}(2 \varepsilon r)^{k},
$$

where $N^{\prime \prime \prime}$ is a constant. On the other hand, the confluent hyper-geometric functions is defined as a series expansion [48]

$$
{ }_{1} F_{1}(-n, \gamma, s)=\sum_{j=0}^{n} \frac{(-1)^{j} n !}{(n-j) ! j !} \frac{\Gamma(\gamma)}{\Gamma(\gamma+j)} s^{j} .
$$

So, we find the upper-spinor component of wave function as

$$
F_{n \kappa}(r)=\mathbf{N} e^{-\varepsilon r} r^{v+1 / 2} L_{n}^{2 v}(2 \varepsilon r)=\mathbf{N} \frac{\Gamma(n+2 v+1)}{n ! \Gamma(2 v+1)} e^{-\varepsilon r} r^{v+1 / 2}{ }_{1} F_{1}(-n, 2 v+1,2 \varepsilon r),
$$

where $\mathbf{N}$ is normalization constant. By using the normalization condition given as $\int_{0}^{\infty}\left|F_{n \kappa}(r)\right|^{2} d r=1$, and the relation between the Laguerre polynomials and confluent hypergeometric functions as $L_{n}^{p}(x)=(\Gamma(n+p+1) / n ! \Gamma(p+1)){ }_{1} F_{1}(-n, p+1, x)$ [47], the normalization constant in (3.17) is written as

$$
\mathbf{N}=(2 \varepsilon)^{v+1} \sqrt{\frac{n !}{\Gamma(n+2 v+1)(2 n+2 v+1)}}
$$

where we have used [47]

$$
\int_{0}^{\infty} x^{q+1} e^{-x} L_{n}^{q}(x) L_{n^{\prime}}^{q}(x) d x=(2 n+q+1) \frac{\Gamma(n+q+1)}{n !} \delta_{n n^{\prime}}
$$

Inserting the parameters in (3.5) and (3.6b) into (3.12), one obtain the transcendental energy eigenvalue equation as follows

$$
\begin{array}{r}
\left(m_{0} c^{2}-E_{n \kappa}\right)\left(m_{0} c^{2}+E_{n \kappa}-C_{s}\right)=\frac{1}{4}\left[\frac{q\left(m_{0} c^{2}+E_{n \kappa}-C_{s}\right)+b\left(C_{s}-2 m_{0} c^{2}\right)}{n+1 / 2+\sqrt{(\kappa+T+1 / 2)^{2}+b(b-q)}}\right]^{2}, \\
n=0,1,2, \ldots
\end{array}
$$

which is identical to [18] when $2 q \rightarrow q$ and the tensor interaction is removed, that is, $T=0$. For the constant mass case, we have

$$
\left(m_{0} c^{2}-E_{n \kappa}\right)\left(m_{0} c^{2}+E_{n \kappa}-C_{s}\right)=\frac{1}{4}\left[\frac{q\left(m_{0} c^{2}+E_{n \kappa}-C_{s}\right)}{n+\kappa+T+1}\right]^{2}, \quad n=0,1,2, \ldots
$$


In case when $C_{s}=0, T=0, \kappa=l, \Sigma(r)=V(r), m_{0} c^{2}-E_{n \kappa} \rightarrow-E_{n l}$ and $m_{0} c^{2}+E_{n \kappa} \rightarrow m_{0}$, the above equation reduces to the non-relativistic energy limit (in units of $\hbar=1$ ):

$$
E_{n l}=-\frac{2 m_{0} q^{2}}{(n+l+1)^{2}}
$$

The wave function (3.17) is finite in the entire range and at the boundaries, that is, $F_{n \kappa}(r=$ $0)=0$ and $F_{n \kappa}(r \rightarrow \infty) \rightarrow 0$.

The lower spinor wave function can be obtained via

$$
\begin{aligned}
G_{n \kappa}(r)= & \frac{1}{\left(m_{0} c^{2}+\hbar c b / r+E_{n \kappa}-C_{s}\right)} \\
& \times\left\{\left(-\varepsilon+\frac{v+1 / 2}{r}+\frac{\kappa+T}{r}\right) F_{n \kappa}(r)-2 \mathbf{N} \varepsilon e^{-\varepsilon r} r^{\nu+1 / 2} L_{n-1}^{2 v+1}(2 \varepsilon r)\right\},
\end{aligned}
$$

where we have used

$$
\frac{d}{d x} L_{n}^{\alpha}(x)=-L_{n-1}^{(\alpha+1)}(x)=\frac{1}{x}\left[n L_{n}^{\alpha}(x)-(n+\alpha) L_{n-1}^{\alpha}(x)\right] .
$$

In Tables 1,2 , and 3 with $\hbar=c=1$, we give some numerical results for the energy eigenvalues from energy formula (3.20).

\section{2. p-Spin Symmetry Case}

To avoid repetition in the solution of (2.5j), the negative energy solution for p-spin symmetry can be obtained directly from those of the above positive energy solution for spin symmetry by using the parameter mapping [18]:

$$
\begin{gathered}
F_{n \kappa}(r) \longleftrightarrow G_{n \kappa}(r) ; \quad V(r) \longrightarrow-V(r)(q \longrightarrow-q) ; \quad \kappa+1 \longrightarrow \kappa ; \\
E_{n \kappa} \longrightarrow-E_{n \kappa} ; \quad C_{s} \longleftrightarrow-C_{p s} .
\end{gathered}
$$

Following the previous results with the above transformations, we finally arrive at the transcendental energy equation as

$$
\begin{array}{r}
\left(m_{0} c^{2}+E_{n \kappa}\right)\left(m_{0} c^{2}-E_{n \kappa}+C_{p s}\right)=\frac{1}{4}\left[\frac{q\left(m_{0} c^{2}-E_{n \kappa}+C_{p s}\right)+b\left(C_{p s}+2 m_{0} c^{2}\right)}{n+\sqrt{(\kappa+T-1 / 2)^{2}+b(b+q)}+1 / 2}\right]^{2} \\
n=0,1,2, \ldots
\end{array}
$$


Table 1: The bound state energy eigenvalues of the Coulomb potential under the pseudo-spin symmetry limit for several values of $n$ and $\kappa$ for $m_{0}=5.0 \mathrm{fm}^{-1}, \hbar=c=1, q=1, T=(0,1,2,5)$, and $C_{p s}=-1$.

\begin{tabular}{|c|c|c|c|c|c|c|c|}
\hline \multirow{2}{*}{$\tilde{l}$} & \multirow{2}{*}{$n, \kappa<0$} & \multirow{2}{*}{$(l, j=l+1 / 2)$} & \multirow{2}{*}{$m_{1}$} & \multicolumn{4}{|c|}{$E_{n, \kappa<0}$} \\
\hline & & & & $T=0$ & $T=1$ & $T=2$ & $T=5$ \\
\hline \multirow{4}{*}{1} & \multirow{4}{*}{$1,-2$} & \multirow{4}{*}{$1 p_{3 / 2}$} & 0.0 & -4.86154 & -4.75676 & -4.47059 & -4.86154 \\
\hline & & & 0.2 & -4.80508 & -4.66644 & -4.36211 & -4.80508 \\
\hline & & & 0.4 & -4.74207 & -4.57172 & -4.26086 & -4.74207 \\
\hline & & & 0.5 & -4.70859 & -4.52376 & -4.21286 & -4.70859 \\
\hline \multirow{4}{*}{2} & \multirow{4}{*}{$1,-3$} & \multirow{4}{*}{$1 d_{5 / 2}$} & 0.0 & -4.91089 & -4.86154 & -4.75676 & -4.75676 \\
\hline & & & 0.2 & -4.87335 & -4.80508 & -4.66644 & -4.66644 \\
\hline & & & 0.4 & -4.83043 & -4.74207 & -4.57172 & -4.57172 \\
\hline & & & 0.5 & -4.80716 & -4.70859 & -4.52376 & -4.52376 \\
\hline \multirow{4}{*}{3} & \multirow{4}{*}{$1,-4$} & \multirow{4}{*}{$1 f_{7 / 2}$} & 0.0 & -4.93793 & -4.91089 & -4.86154 & -4.47059 \\
\hline & & & 0.2 & -4.91138 & -4.87335 & -4.80508 & -4.36211 \\
\hline & & & 0.4 & -4.88067 & -4.83043 & -4.74207 & -4.26086 \\
\hline & & & 0.5 & -4.86385 & -4.80716 & -4.70859 & -4.21286 \\
\hline \multirow{4}{*}{4} & \multirow{4}{*}{$1,-5$} & \multirow{4}{*}{$1 g_{9 / 2}$} & 0.0 & -4.95431 & -4.93793 & -4.91089 & -4.47059 \\
\hline & & & 0.2 & -4.93461 & -4.91138 & -4.87335 & -4.36211 \\
\hline & & & 0.4 & -4.91166 & -4.88067 & -4.83043 & -4.26086 \\
\hline & & & 0.5 & -4.89903 & -4.86385 & -4.80716 & -4.21286 \\
\hline \multirow{4}{*}{1} & \multirow{4}{*}{$2,-2$} & \multirow{4}{*}{$2 p_{3 / 2}$} & 0.0 & -4.91089 & -4.86154 & -4.75676 & -4.91089 \\
\hline & & & 0.2 & -4.87402 & -4.80794 & -4.69083 & -4.87402 \\
\hline & & & 0.4 & -4.83243 & -4.75015 & -4.62526 & -4.83243 \\
\hline & & & 0.5 & -4.81014 & -4.72024 & -4.59287 & -4.81014 \\
\hline \multirow{4}{*}{2} & \multirow{4}{*}{$2,-3$} & \multirow{4}{*}{$2 d_{5 / 2}$} & 0.0 & -4.93793 & -4.91089 & -4.86154 & -4.86154 \\
\hline & & & 0.2 & -4.9116 & -4.87402 & -4.80794 & -4.80794 \\
\hline & & & 0.4 & -4.88134 & -4.83243 & -4.75015 & -4.75015 \\
\hline & & & 0.5 & -4.86487 & -4.81014 & -4.72024 & -4.72024 \\
\hline \multirow{4}{*}{3} & \multirow{4}{*}{$2,-4$} & \multirow{4}{*}{$2 f_{7 / 2}$} & 0.0 & -4.95431 & -4.93793 & -4.91089 & -4.75676 \\
\hline & & & 0.2 & -4.93469 & -4.9116 & -4.87402 & -4.69083 \\
\hline & & & 0.4 & -4.91194 & -4.88134 & -4.83243 & -4.62526 \\
\hline & & & 0.5 & -4.89945 & -4.86487 & -4.81014 & -4.59287 \\
\hline \multirow{4}{*}{4} & \multirow{4}{*}{$2,-5$} & & 0.0 & -4.96498 & -4.95431 & -4.93793 & -4.75676 \\
\hline & & $2 g_{0}$ & 0.2 & -4.94984 & -4.93469 & -4.91160 & -4.69083 \\
\hline & & $289 / 2$ & 0.4 & -4.93218 & -4.91194 & -4.88134 & -4.62526 \\
\hline & & & 0.5 & -4.92244 & -4.89945 & -4.86487 & -4.59287 \\
\hline
\end{tabular}

which is identical to [18] when $2 q \rightarrow q$ and the tensor interaction is removed, that is, $T=0$. For the constant mass case, we have

$$
\left(m_{0} c^{2}+E_{n \kappa}\right)\left(m_{0} c^{2}-E_{n \kappa}+C_{p s}\right)=\frac{1}{4}\left[\frac{q\left(m_{0} c^{2}-E_{n \kappa}+C_{p s}\right)}{n+\kappa+T}\right]^{2}, \quad n=0,1,2, \ldots,
$$

the lower-spinor component of wave function as

$$
G_{n \kappa}(r)=\tilde{\mathbf{N}} e^{-\tilde{\varepsilon} r} r^{\nu+1 / 2} L_{n}^{2 v}(2 \tilde{\varepsilon} r)=\tilde{\mathbf{N}} \frac{\Gamma(n+2 \tilde{\mathcal{v}}+1)}{n ! \Gamma(2 \widetilde{\mathcal{v}}+1)} e^{-\tilde{\varepsilon} r} r_{1}^{\tilde{\nu}+1 / 2} F_{1}(-n, 2 \tilde{\mathcal{v}}+1,2 \tilde{\varepsilon} r)
$$


Table 2: The bound state energy eigenvalues of the Coulomb potential under the pseudo-spin symmetry limit for several values of $n$ and $\kappa$ for $m_{0}=5.0 \mathrm{fm}^{-1}, q=1, \hbar=c=1, T=(0,1,2,5)$, and $C_{p s}=-1$.

\begin{tabular}{|c|c|c|c|c|c|c|c|}
\hline \multirow{2}{*}{$\tilde{l}$} & \multirow{2}{*}{$n-1, \kappa>0$} & \multirow{2}{*}{$(l, j=l-1 / 2)$} & \multirow{2}{*}{$m_{1}$} & \multicolumn{4}{|c|}{$E_{n-1, \kappa>0}$} \\
\hline & & & & $T=0$ & $T=1$ & $T=2$ & $T=5$ \\
\hline \multirow{4}{*}{2} & \multirow{4}{*}{0,2} & \multirow{4}{*}{$0 d_{3 / 2}$} & 0.0 & -4.75676 & -4.86154 & -4.91089 & -4.96498 \\
\hline & & & 0.2 & -4.66644 & -4.80508 & -4.87335 & -4.94979 \\
\hline & & & 0.4 & -4.57172 & -4.74207 & -4.83043 & -4.93205 \\
\hline & & & 0.5 & -4.52376 & -4.70859 & -4.80716 & -4.92225 \\
\hline \multirow{4}{*}{3} & \multirow{4}{*}{0,3} & \multirow{4}{*}{$0 f_{5 / 2}$} & 0.0 & -4.86154 & -4.91089 & -4.93793 & -4.97231 \\
\hline & & & 0.2 & -4.80508 & -4.87335 & -4.91138 & -4.96026 \\
\hline & & & 0.4 & -4.74207 & -4.83043 & -4.88067 & -4.94614 \\
\hline & & & 0.5 & -4.70859 & -4.80716 & -4.86385 & -4.93833 \\
\hline \multirow{4}{*}{4} & \multirow{4}{*}{0,4} & \multirow{4}{*}{$0 g_{7 / 2}$} & 0.0 & -4.91089 & -4.93793 & -4.95431 & -4.97756 \\
\hline & & & 0.2 & -4.87335 & -4.91138 & -4.93461 & -4.96777 \\
\hline & & & 0.4 & -4.83043 & -4.88067 & -4.91166 & -4.95628 \\
\hline & & & 0.5 & -4.80716 & -4.86385 & -4.89903 & -4.94991 \\
\hline \multirow{4}{*}{5} & \multirow{4}{*}{0,5} & \multirow{4}{*}{$0 h_{9 / 2}$} & 0.0 & -4.93793 & -4.95431 & -4.96498 & -4.98144 \\
\hline & & & 0.2 & -4.91138 & -4.93461 & -4.94979 & -4.97334 \\
\hline & & & 0.4 & -4.88067 & -4.91166 & -4.93205 & -4.96381 \\
\hline & & & 0.5 & -4.86385 & -4.89903 & -4.92225 & -4.95852 \\
\hline \multirow{4}{*}{2} & \multirow{4}{*}{1,2} & \multirow{4}{*}{$1 d_{3 / 2}$} & 0.0 & -4.86154 & -4.91089 & -4.93793 & -4.97231 \\
\hline & & & 0.2 & -4.80794 & -4.87402 & -4.91160 & -4.96028 \\
\hline & & & 0.4 & -4.75015 & -4.83243 & -4.88134 & -4.94621 \\
\hline & & & 0.5 & -4.72024 & -4.81014 & -4.86487 & -4.93843 \\
\hline \multirow{4}{*}{3} & \multirow{4}{*}{1,3} & \multirow{4}{*}{$1 f_{5 / 2}$} & 0.0 & -4.91089 & -4.93793 & -4.95431 & -4.97756 \\
\hline & & & 0.2 & -4.87402 & -4.91160 & -4.93469 & -4.96778 \\
\hline & & & 0.4 & -4.83243 & -4.88134 & -4.91194 & -4.95632 \\
\hline & & & 0.5 & -4.81014 & -4.86487 & -4.89945 & -4.94997 \\
\hline \multirow{4}{*}{4} & \multirow{4}{*}{1,4} & \multirow{4}{*}{$1 g_{7 / 2}$} & 0.0 & -4.93793 & -4.95431 & -4.96498 & -4.98144 \\
\hline & & & 0.2 & -4.91160 & -4.93469 & -4.94984 & -4.97757 \\
\hline & & & 0.4 & -4.88134 & -4.91194 & -4.93218 & -4.96383 \\
\hline & & & 0.5 & -4.86487 & -4.89945 & -4.92244 & -4.95856 \\
\hline \multirow{4}{*}{5} & \multirow{4}{*}{1,5} & & 0.0 & -4.95431 & -4.96498 & -4.97231 & -4.98440 \\
\hline & & $1 h_{9 / 2}$ & 0.2 & -4.93469 & -4.94984 & -4.96028 & -4.97758 \\
\hline & & $17 t 9 / 2$ & 0.4 & -4.91194 & -4.93218 & -4.94621 & -4.96957 \\
\hline & & & 0.5 & -4.89945 & -4.92244 & -4.93843 & -4.96512 \\
\hline
\end{tabular}

where $\widetilde{\varepsilon}^{2}=\left(1 /(\hbar c)^{2}\right)\left(m_{0} c^{2}+E_{n \kappa}\right)\left(m_{0} c^{2}-E_{n \kappa}+C_{p s}\right), \widetilde{v}^{2}=(\kappa+T-1 / 2)^{2}+b(b+q)$ and $\tilde{\mathbf{N}}$ is normalization constant

$$
\tilde{\mathbf{N}}=(2 \widetilde{\varepsilon})^{\tilde{v}+1} \sqrt{\frac{n !}{\Gamma(n+2 \tilde{v}+1)(2 n+2 \widetilde{v}+1)}}
$$

The upper-spinor wave function can be obtained via

$$
\begin{aligned}
F_{n \kappa}(r)= & \frac{1}{\left(m_{0} c^{2}+\hbar c b / r-E_{n \mathcal{K}}+C_{p s}\right)} \\
& \times\left\{\left(-\widetilde{\varepsilon}+\frac{\tilde{v}+1 / 2}{r}-\frac{\kappa+T}{r}\right) G_{n \kappa}(r)-2 \tilde{\mathbf{N}} \tilde{\varepsilon} e^{-\tilde{\varepsilon} r} r^{\tilde{v}+1 / 2} L_{n-1}^{2 \tilde{v}+1}(2 \widetilde{\varepsilon} r)\right\} .
\end{aligned}
$$


Table 3: The bound state energy eigenvalues of the Coulomb potential for several states under the pseudospin symmetry limit with values of $C_{p s}$ with $m_{0}=5.0 \mathrm{fm}^{-1}, q=1, \hbar=c=1, m_{1}=(0,0.5)$, and $T=(0,5)$.

\begin{tabular}{|c|c|c|c|c|c|c|c|}
\hline \multirow{3}{*}{$\tilde{l}$} & \multirow{3}{*}{$n, \kappa$} & \multirow{3}{*}{$(l, j=l+1 / 2)$} & \multirow{3}{*}{$C_{p s}$} & \multicolumn{4}{|c|}{$E_{n, \kappa<0}$} \\
\hline & & & & $T=0$ & $T=0$ & $T=5$ & $T=5$ \\
\hline & & & & $m_{1}=0$ & $m_{1}=0.5$ & $m_{1}=0$ & $m_{1}=0.5$ \\
\hline \multirow{8}{*}{1} & \multirow{8}{*}{1,2} & \multirow{8}{*}{$1 d_{3 / 2}$} & -1.00 & -4.75676 & -4.52376 & -4.96498 & -4.92225 \\
\hline & & & -1.25 & -4.76351 & -4.53699 & -4.96595 & -4.92441 \\
\hline & & & -1.50 & -4.77027 & -4.55022 & -4.96693 & -4.92657 \\
\hline & & & -1.75 & -4.77703 & -4.56345 & -4.96790 & -4.92873 \\
\hline & & & -2.00 & -4.78378 & -4.57668 & -4.96887 & -4.93089 \\
\hline & & & -2.25 & -4.79054 & -4.58991 & -4.96984 & -4.93305 \\
\hline & & & -2.50 & -4.79730 & -4.60313 & -4.97082 & -4.93521 \\
\hline & & & -2.75 & -4.80405 & -4.61636 & -4.97179 & -4.93736 \\
\hline \multirow{8}{*}{3} & \multirow{8}{*}{$0,-4$} & \multirow{8}{*}{$0 f_{7 / 2}$} & -1.00 & -4.91089 & -4.80543 & -3.20000 & -2.90681 \\
\hline & & & -1.25 & -4.91337 & -4.81083 & -3.25000 & -2.96496 \\
\hline & & & -1.50 & -4.91584 & -4.81624 & -3.30000 & -3.0231 \\
\hline & & & -1.75 & -4.91832 & -4.82164 & -3.35000 & -3.08124 \\
\hline & & & -2.00 & -4.92079 & -4.82705 & -3.40000 & -3.13939 \\
\hline & & & -2.25 & -4.92327 & -4.83245 & -3.45000 & -3.19753 \\
\hline & & & -2.50 & -4.92574 & -4.83786 & -3.50000 & -3.25568 \\
\hline & & & -2.75 & -4.92822 & -4.84326 & -3.55000 & -3.31382 \\
\hline \multirow{8}{*}{1} & \multirow{8}{*}{$1,-2$} & \multirow{8}{*}{$1 p_{3 / 2}$} & -1.00 & -4.86154 & -4.70859 & -4.86154 & -4.70859 \\
\hline & & & -1.25 & -4.86538 & -4.71669 & -4.86538 & -4.71669 \\
\hline & & & -1.50 & -4.86923 & -4.72478 & -4.86923 & -4.72478 \\
\hline & & & -1.75 & -4.87308 & -4.73288 & -4.87308 & -4.73288 \\
\hline & & & -2.00 & -4.87692 & -4.74097 & -4.87692 & -4.74097 \\
\hline & & & -2.25 & -4.88077 & -4.74907 & -4.88077 & -4.74907 \\
\hline & & & -2.50 & -4.88462 & -4.75716 & -4.88462 & -4.75716 \\
\hline & & & -2.75 & -4.88846 & -4.76526 & -4.88846 & -4.76526 \\
\hline \multirow{8}{*}{3} & \multirow{8}{*}{$1,-4$} & \multirow{8}{*}{$1 f_{7 / 2}$} & -1.00 & -4.93793 & -4.86385 & -4.47059 & -4.21286 \\
\hline & & & -1.25 & -4.93966 & -4.86764 & -4.48529 & -4.23472 \\
\hline & & & -1.50 & -4.94138 & -4.87142 & -4.50000 & -4.25659 \\
\hline & & & -1.75 & -4.9431 & -4.8752 & -4.51471 & -4.27845 \\
\hline & & & -2.00 & -4.94483 & -4.87898 & -4.52941 & -4.30032 \\
\hline & & & -2.25 & -4.94655 & -4.88276 & -4.54412 & -4.32218 \\
\hline & & & -2.50 & -4.94828 & -4.88654 & -4.55882 & -4.34405 \\
\hline & & & -2.75 & -4.95000 & -4.89033 & -4.57353 & -4.36591 \\
\hline
\end{tabular}

\section{Numerical Results}

In Tables 1 to 3 , we see that energies of bound states such as: $\left(n p_{1 / 2}, n p_{3 / 2}\right),\left(n d_{3 / 2}, n d_{5 / 2}\right)$, $\left(n f_{5 / 2}, n f_{7 / 2}\right),\left(n g_{7 / 2}, n g_{9 / 2}\right), \ldots$ (where each pair is considered as a spin doublet) in the absence of the tensor potential are degenerate but in the presence of the tensor potential, the degeneracies are removed. Also, we investigate the effects of the $m_{1}$ and $C_{s}$ parameters on the bound states under the conditions of the spin symmetry limits for $T=0$. The results are given in Tables 1 to 3 . It is readily seen that if $m_{1}$ and $C_{s}$ parameters increases, the value of the bound state energy eigenvalues of this potential increases for several states. It is shown 


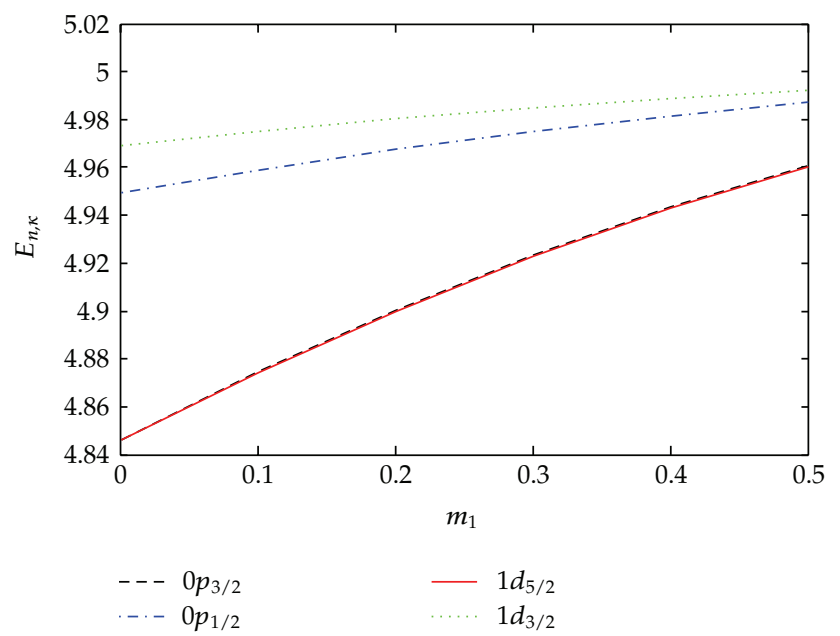

Figure 1: The variation of the energy levels as a function $m_{1}$ in the presence of spin symmetry.

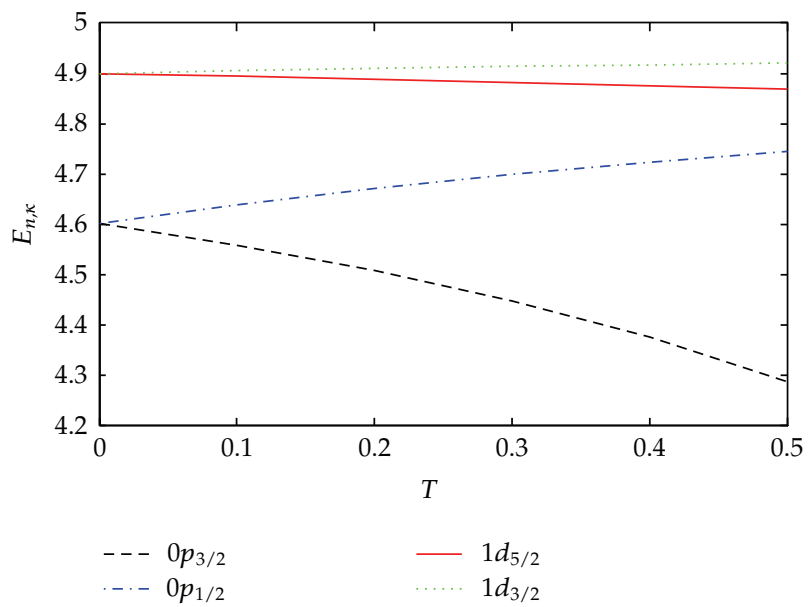

Figure 2: The variation of the energy levels as a function $T$ in the presence of spin symmetry.

that the energy eigenvalues decrease with decreasing $m_{1}$ and when increase with increasing the tensor strength $T$ for $\kappa>0$ and $\kappa<0$. In Table 3, for constant values of $C_{s}$ and $T$, the energy increases when $m_{1}$ increasing. The decrease in the energy values is large without tensor interaction while small in presence of tensor interaction and then being large. Further, when $C_{s}$ increases, the energy increasing.

Finally, we plot the relativistic energy eigenvalues under spin and p-spin symmetry limitations in Figures 1 to 4. In Figure 2, we plot the energy eigenvalues of spin symmetry limit versus the perturbated mass $m_{1}$. It is seen that when $m_{1}$ increases, the energy increases too. In Figure 2, we have shown the variation of the energy as a function of $T$. We can see the degeneracy removes between spin doublets and also they become far from each other, when the parameter $T$ increases. In Figures 3 to 4 , we plot the energy states of the pseudospin symmetry limit for different levels as functions of parameters $m_{1}$ and $T$, respectively. The variation of energy can also be seen from these figures. 


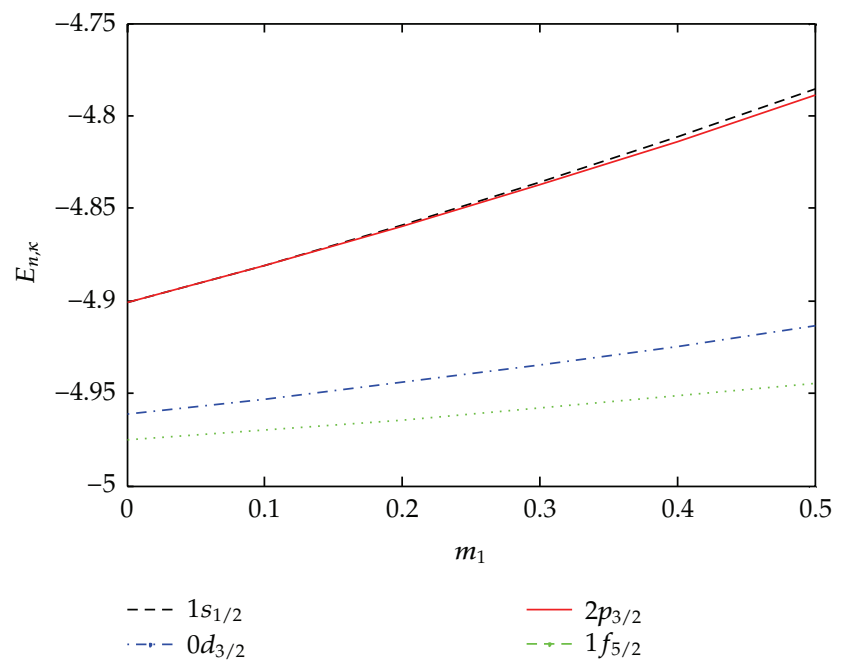

Figure 3: The variation of the energy levels as a function $m_{1}$ in the presence of pseudospin symmetry.

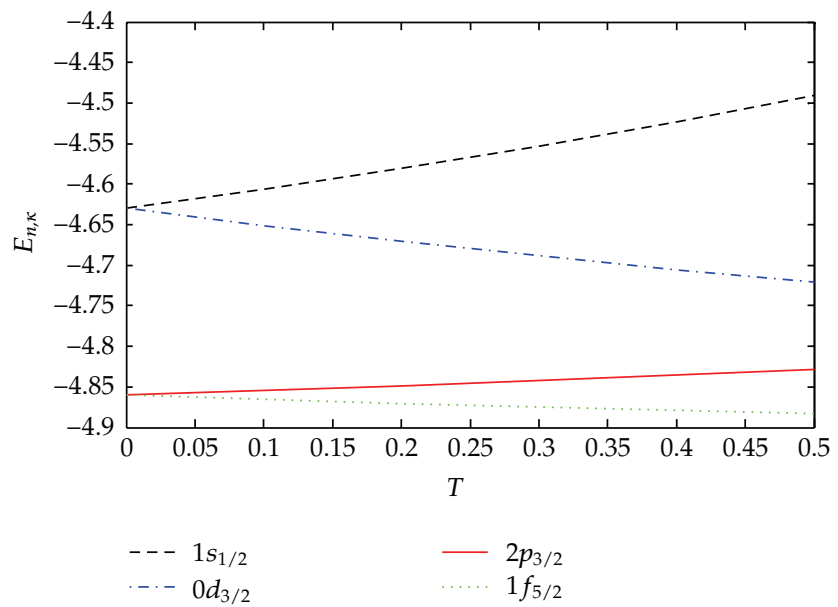

Figure 4: The variation of the energy levels as a function $T$ in the presence of pseudospin symmetry.

\section{Conclusion}

In this paper, the relativistic equation for particles with spin $1 / 2$ was solved exactly with both spatially-dependent mass and tensor interaction for attractive scalar and repulsive vector Coulomb potentials under the spin symmetry limit via the Laplace transformation method. Some numerical results are given for specific values of the model parameters. Effects of the tensor interaction on the bound states were presented that tensor interaction removes degeneracy between two states in spin doublets. We also investigated the effects of the spatially-dependent mass on the bound states under the conditions of the spin symmetry limits for $T=0$. 


\section{References}

[1] I. C. Wang and C. Y. Wong, "Finite-size effect in the Schwinger particle-production mechanism," Physical Review D, vol. 38, pp. 348-359, 1988.

[2] G. Mao, "Effect of tensor couplings in a relativistic Hartree approach for finite nuclei," Physical Review C, vol. 67, Article ID 044318, 12 pages, 2003.

[3] R. J. Furnstahl, J. J. Rusnak, and B. D. Serot, "The nuclear spin-orbit force in chiral effective field theories," Nuclear Physics A, vol. 632, no. 4, pp. 607-623, 1998.

[4] P. Alberto, R. Lisboa, M. Malheiro, and A. S. de Castro, "Tensor coupling and pseudospin symmetry in nuclei," Physical Review C, vol. 71, Article ID 034313, 7 pages, 2005.

[5] Y. I. Salamin, S. X. Hu, K. Z. Hatsagortsyan, and C. H. Keitel, "Relativistic high-power laser-matter interactions," Physics Reports, vol. 427, no. 2-3, pp. 41-155, 2006.

[6] M. I. Katsnelson, K. S. Novoselov, and A. K. Geim, "Chiral tunnelling and the Klein paradox in graphene," Nature Physics, vol. 2, no. 9, pp. 620-625, 2006.

[7] M. R. Galler and W. Kohn, "Quantum mechanics of electrons in crystals with graded composition," Physical Review Letters, vol. 70, pp. 3103-3106, 1993.

[8] A. Puente and M. Gasas, "Non-local energy density functional for atoms and metal clusters," Computational Materials Science, vol. 2, pp. 441-449, 1994.

[9] F. Arias De Saavedra, J. Boronat, A. Polls, and A. Fabrocini, "Effective mass of one ${ }^{4} \mathrm{He}$ atom in liquid ${ }^{3} \mathrm{He}^{\prime}$ Physical Review B, vol. 50, no. 6, pp. 4248-4251, 1994.

[10] M. Barranco, M. Pi, S. M. Gatica, E. S. Hernandez, and J. Navarro, "Structure and energetics of mixed ${ }^{4}$ He- ${ }^{3}$ He drops," Physical Review B, vol. 56, pp. 8997-9003, 1997.

[11] A. Puente, L. I. Serra, and M. Casas, "Dipole excitation of Na clusters with a non-local energy density functional," Zeitschrift Für Physik D, vol. 31, pp. 283-286, 1994.

[12] L. Serra and E. Lipparini, "Spin response of unpolarized quantum dots," Europhysics Letters, vol. 40, no. 6, pp. 667-672, 1997.

[13] G. Bastard, Wave Mechanics Applied to Semiconductor Heterostructures, Les Editions de physique, Les Ulis, France, 1988.

[14] A. G. M. Schmidt, "Wave-packet revival for the Schrödinger equation with position-dependent mass," Physics Letters A, vol. 353, pp. 459-462, 2006.

[15] M. Eshghi and H. Mehraban, "Solution of the dirac equation with position-dependent mass for qparameter modified pöschl-teller and coulomb-like tensor potential," Few-Body Systems, vol. 52, pp. 41-47, 2012.

[16] E. Maghsoodi, H. Hassanabadi, and S. Zarrinkamar, "Spectrum of dirac equation under deng-fan scalar and vector potentials and a coulomb tensor interaction by SUSYQM," Few-Body Systems, vol. 53, no. 3-4, pp. 525-538, 2012.

[17] C. S. Jia and A. de Souza Dutra, "Extension of PT-symmetric quantum mechanics to the Dirac theory with position-dependent mass," Annals of Physics, vol. 323, no. 3, pp. 566-579, 2008.

[18] S. M. Ikhdair and R. Sever, "Solutions of the spatially-dependent mass Dirac equation with the spin and pseudospin symmetry for the Coulomb-like potential," Applied Mathematics and Computation, vol. 216, no. 2, pp. 545-555, 2010.

[19] Y. Xu, S. He, and C.-S. Jia, "Approximate analytical solutions of the Dirac equation with the PöschlTeller potential including the spin-orbit coupling term," Journal of Physics A, vol. 41, no. 25, p. 255302, 8, 2008.

[20] H. Akcay, "Dirac equation with scalar and vector quadratic potentials and Coulomb-like tensor potential," Physics Letters A, vol. 373, no. 6, pp. 616-620, 2009.

[21] M. Eshghi and M. Hamzavi, "Spin symmetry in dirac-attractive radial problem and tensor potential," Communications in Theoretical Physics, vol. 57, article 355, 2012.

[22] M. Eshghi, "Pseudo-harmonic oscillatory ring-shaped potential in a relativistic equation," Chinese Physics Letters, vol. 29, no. 11, Article ID 110304, 2012.

[23] O. Panella, S. Biondini, and A. Arda, "New exact solution of the one-dimensional Dirac equation for the Woods-Saxon potential within the effective mass case," Journal of Physics A, vol. 43, Article ID 325302, 2010.

[24] A. D. Alhaidari, "Solution of the Dirac equation with position-dependent mass in the Coulomb field," Physics Letters A, vol. 322, no. 1-2, pp. 72-77, 2004.

[25] X.-L. Peng, J.-Y. Liu, and C.-S. Jia, "Approximation solution of the Dirac equation with positiondependent mass for the generalized Hulthén potential," Physics Letters A, vol. 352, no. 6, pp. 478-483, 2006. 
[26] M. Eshghi and H. Mehraban, "Eigen spectra for Manning-Rosen potential including a Coulomb-like tensor interaction," International Journal of Physical Sciences, vol. 6, no. 29, pp. 6643-6652, 2011.

[27] M. Hamzavi, M. Eshghi, and S. M. Ikhdair, "Effect of tensor interaction in the Dirac-attractive radial problem under pseudospin symmetry limit," Journal of Mathematical Physics, vol. 53, Article ID 082101, 10 pages, 2012.

[28] M.-C. Zhang and G.-Q. Huang-Fu, "Solution of the Dirac equation in the tridiagonal representation with pseudospin symmetry for an anharmonic oscillator and electric dipole ring-shaped potential," Annals of Physics, vol. 327, no. 3, pp. 841-850, 2012.

[29] O. Aydogdu and R. Sever, "Exact solution of the Dirac equation with the Mie-type potential under the pseudospin and spin symmetry limit," Annals of Physics, vol. 325, pp. 373-383, 2010.

[30] M. Eshghi and H. Mehraban, "Eigen spectra in the Dirac-hyperbolic problem with tensor coupling," Chinese Journal of Physics, vol. 50, no. 4, p. 533, 2012.

[31] M. Eshghi, "Dirac-hyperbolic scarf problem including a coulomb-like tensor potential," Acta Scientiarum, vol. 34, p. 207, 2012.

[32] G. F. Wei and S. H. Dong, "A novel algebraic approach to spin symmetry for Dirac equation with scalar and vector second Pöschl-Teller potentials," The European Physical Journal A, vol. 43, pp. 185-190, 2010.

[33] V. G. C. S. dos Santos, A. de Souza Dutra, and M. B. Hott, "Real spectra for the non-Hermitian Dirac equation in $1+1$ dimensions with the most general coupling," Physics Letters A, vol. 373, no. 38, pp. 3401-3406, 2009.

[34] M. Hamzavi, A. A. Rajabi, and H. Hassanabadi, "Exact spin and pseudospin symmetry solutions of the Dirac equation for mie-type potential including a coulomb-like tensor potential," Fezw-Body Systems, vol. 48, no. 2, pp. 171-182, 2010.

[35] G. Doetsch, Guide to the Applications of Laplace Transforms, Princeton University press, Princeton, NJ, USA, 1961.

[36] G. Chen, "The exact solutions of the Schrödinger equation with the Morse potential via Laplace transforms," Physics Letters A, vol. 326, no. 1-2, pp. 55-57, 2004.

[37] A. Arda and R. Sever, "Exact solutions of the Schrödinger equation via Laplace transform approach: pseudoharmonic potential and Mie-type potentials," Journal of Mathematical Chemistry, vol. 50, no. 4, pp. 971-980, 2012.

[38] E. Schrodinger, "Quantisierung als Eigenwertproblem. I and II," Annalen Der Physik, vol. 79, pp. 361376, 1926.

[39] R. A. Swainson and G. W. F. Drake, "A unified treatment of the nonrelativistic and relativistic hydrogen atom. I. The wavefunctions," Journal of Physics A, vol. 24, no. 1, pp. 79-94, 1991.

[40] G. Chen, "Exact solutions of $N$-dimensional harmonic oscillator via Laplace transformation," Chinese Physics, vol. 14, no. 6, p. 1075, 2005.

[41] J. D. Bjorken and S. D. Drell, Relativistic Quantum Mechanics, McGraw-Hill, New York, NY, USA, 1964.

[42] D. Agboola, "Dirac-Hulthen Problem with Position-dependent Mass in D-dimensions," http://arxiv .org/abs/1011.2368.

[43] A. Arda, R. Sever, and C. Tezcan, "Approximate analytical solutions of the effective mass Dirac equation for the generalized Hulthén potential with any $\mathcal{\kappa}$-value," Central European Journal of Physics, vol. 8, no. 5, pp. 843-849, 2010.

[44] J. Meng, K. Sugawara-Tanabe, S. Yamaji, P. Ring, and A. Arima, "Pseudospin symmetry in relativistic mean field theory," Physical Review C, vol. 58, no. 2, pp. R628-R631, 1998.

[45] J. Meng, K. Sugawara-Tanaha, S. Yamaji, and A. Arima, "Pseudospin symmetry in Zr and Sn isotopes from the proton drip line to the neutron drip line," Physical Review C, vol. 59, pp. 154-163, 1999.

[46] M. R. Spiegel, Schaum's Outline of Theory and Problems of Laplace Transforms, Schaum Publishing, New York, NY, USA, 1965.

[47] F. Doetsch, Guides to the Application of Laplace Transforms, Princeton University press, Princeton, NJ, USA, 1961.

[48] M. Abramowitz and I. A. Stegun, Handbook Mathematical Functions With Formulas, Graphs, and Mathematical Tables, Dover, New York, NY, USA, 1970. 

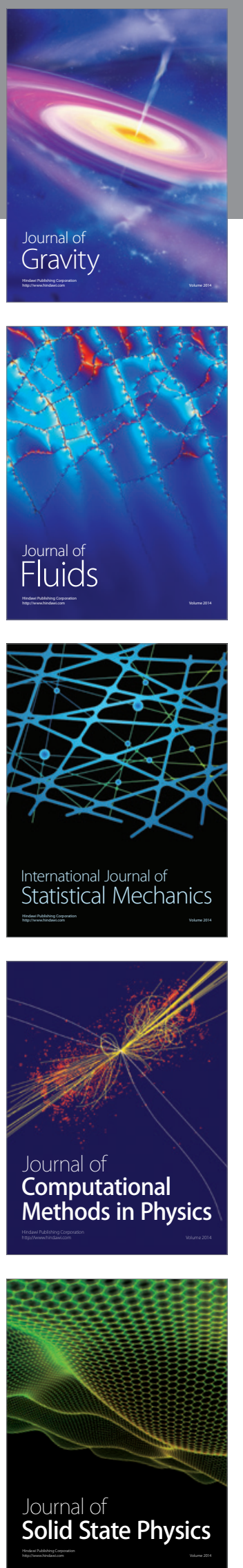

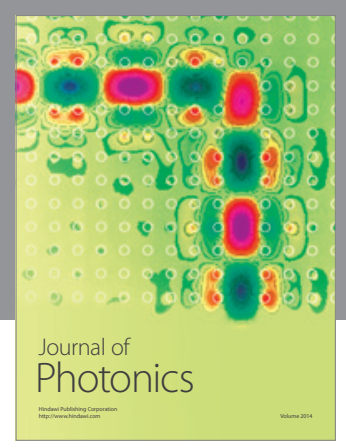

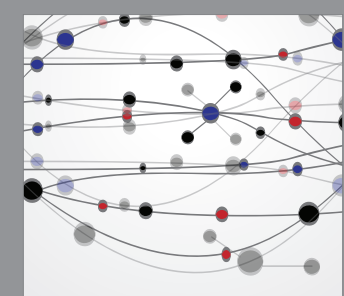

The Scientific World Journal
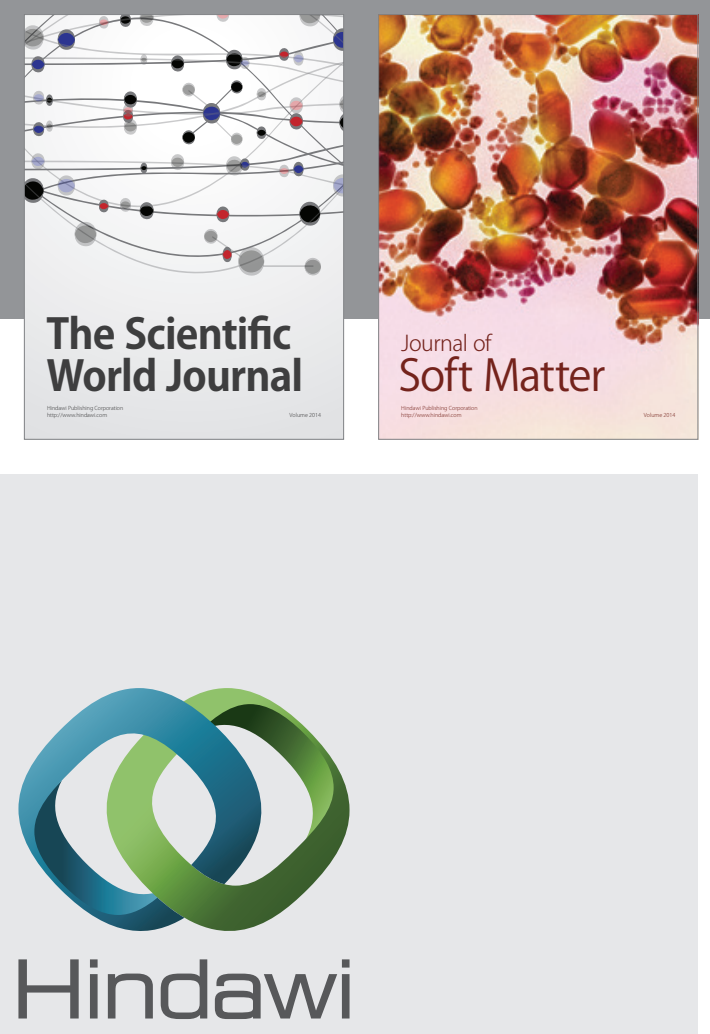

Submit your manuscripts at

http://www.hindawi.com
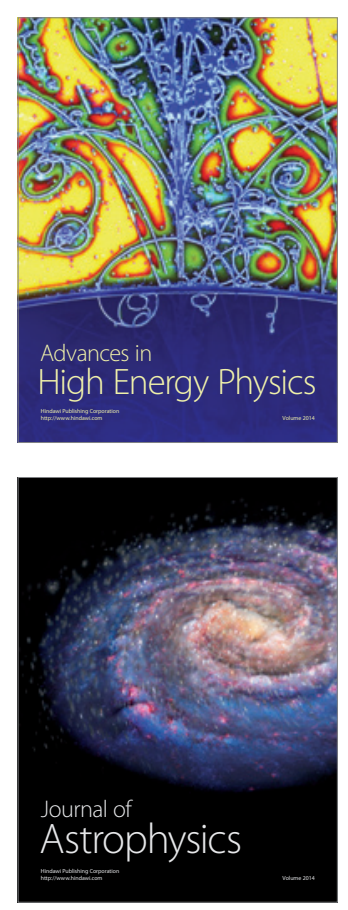
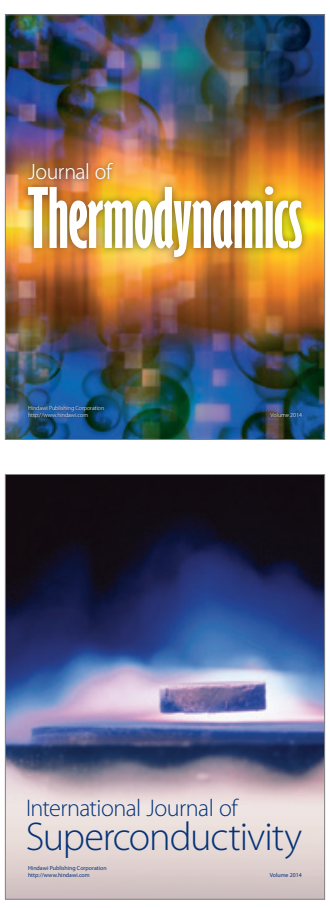
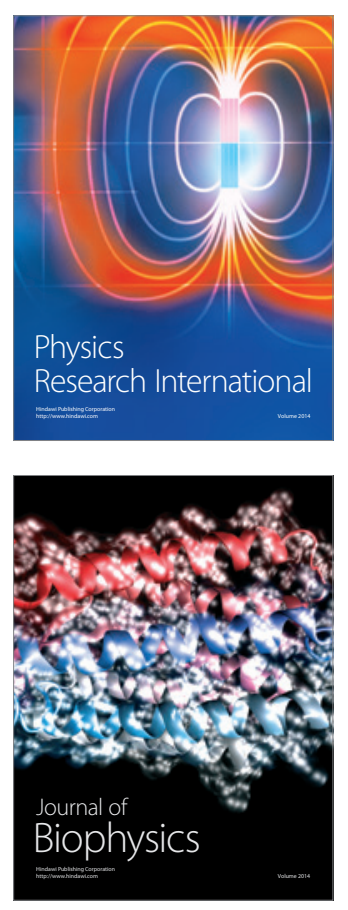
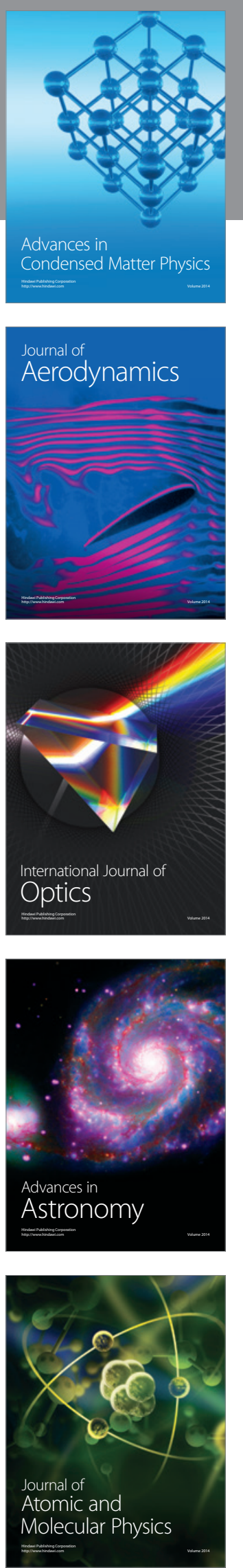